\title{
THE TECHNIQUES AND PROGRESS OF WIND AND TEMPERATURE LIDAR IN WIPM
}

\author{
Faquan Li ${ }^{1 *}$, Yong Yang ${ }^{1,2}$, Xuewu Cheng ${ }^{1}$, Yajuan $\mathrm{Li}^{1,3}$, Xin Lin ${ }^{1}$, Yuan Xia ${ }^{1,3}$, Linmei Liu ${ }^{1}$, Shalei \\ Song $^{1}$, Zhenwei Chen ${ }^{1}$, Jun Xiong ${ }^{1,3}$, Kuijun Wu ${ }^{1,3}$, Shunsheng Gong ${ }^{1}$
}

\author{
${ }^{1}$ State Key Laboratory of Magnetic Resonance and Atomic and Molecular Physics, Wuhan Institute of \\ Physics and Mathematics, Chinese Academy of Sciences, Wuhan 430071, China,
}

*Email: lifaquan@wipm.ac.cn

2 Department of Atmospheric and Planetary Sciences, Center for Atmospheric Sciences, Hampton University, Hampton, Virginia 23668, USA,

\section{University of Chinese Academy of Sciences, Beijing 100049, China}

\begin{abstract}
In this paper, a lidar system in Wuhan Institute of Physics and Mathematics (WIPM, $30.5^{\circ} \mathrm{N}, 114.5^{\circ} \mathrm{E}$ ) for atmospheric density, temperature and wind observation was reported. The design and techniques of this lidar were described in detail. The atmospheric temperature of the troposphere, stratosphere and lower mesosphere were measured by the Raman, Rayleigh and sodium channel of this lidar system, respectively.
\end{abstract}

\section{INTRODUCTION}

The vertical profiles of atmospheric density, temperature and wind describe the variation of atmospheric phenomena directly. They are key parameters for the research and application of atmospheric dynamics and thermodynamics $[1,2]$.

The lidar (light detection and range) was developed to observe the atmospheric density, temperature and wind with high spatial resolution, high temporal resolution and high spectral resolution. The Rayleigh lidar measure the temperature of the stratosphere and mesosphere over altitudes of $\sim 35 \mathrm{~km}$ to $\sim 80 \mathrm{~km}[3]$. The altitude lower limit for a Rayleigh temperature lidar is decided by the aerosol in the lower atmosphere. The vibrational and pure rotational Raman lidar which is based on the pure molecular scattering without Mie scattering by aerosol particles, is developed to measure the atmosphere from near ground to the stratosphere[4-7]. The altitude upper limit for the Rayleigh temperature lidar depends on the atmospheric molecular number density and lidar parameters, especially the laser power and effective area of the optical receivers. The metal resonance fluorescence lidar is employed to observe the mesosphere and lower thermosphere, since the effective cross-section of the metal atoms in the mesopause is several orders of magnitude larger than that of the Rayleigh scattering[8-10]. The sodium lidar is one of the most popular metal resonance fluorescence lidars due to its high population and large cross section[11, 12]. The Rayleigh lidar, Raman lidar and potassium resonance fluorescence lidar were combined to observe the whole profile of the atmospheric temperature from 1 to $105 \mathrm{~km}[13]$.

In this paper, a lidar system for atmospheric observation was reported. This lidar system was designed to observe the vertical profiles of the atmospheric density, temperature and wind. The Rotational Raman channel, Rayleigh temperature channel and sodium resonance fluorescence channel of this lidar system were employed to measure the temperature of the troposphere, stratosphere and mesopause respectively. The design and progress of this system were described in section 2. In section 3, the observational results of temperature profile was discussed.

\section{METHODOLOGY}

As a part of the APSOS (Atmospheric Profiling Synthetic Observation System) project granted by the NSFC (National natural Science Foundation of China), a new lidar system is designed and under construction aiming to investigate the neutral atmospheric dynamics and thermodynamic progress, especially in the tropopause, stratopause and mesopause area. Compared with the old lidar system in WIPM (Wuhan Institute of Physics and Mathematics)[14], the pulsed lasers of the transmitter in the new lidar are narrow band with single mode, and therefore more atmospheric 
parameters will be measured by this new system, especially the wind and the temperature in the troposphere and mesopause. This lidar system includes the transmitter, optical receiver and signal and data platform, as shown in figure 1 .

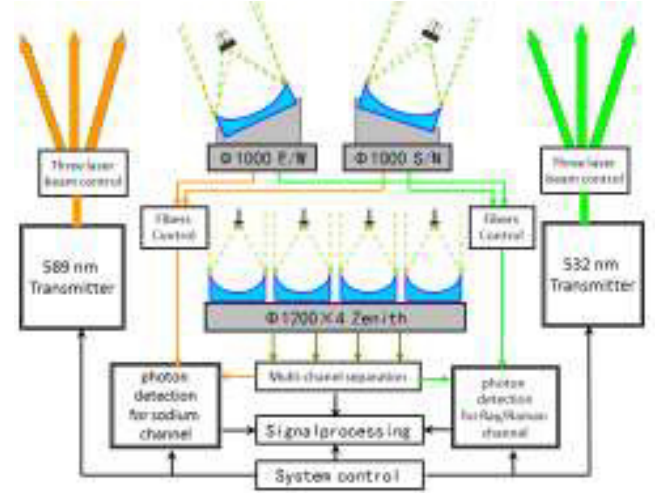

Figure 1 the schematic of the lidar system

The transmitter includes a $589 \mathrm{~nm}$ yellow laser and a $532 \mathrm{~nm}$ green laser, as shown in figure 2 . The frequency of the $532 \mathrm{~nm}$ laser is locked by the absorption spectrum of the iodine line 1109 .

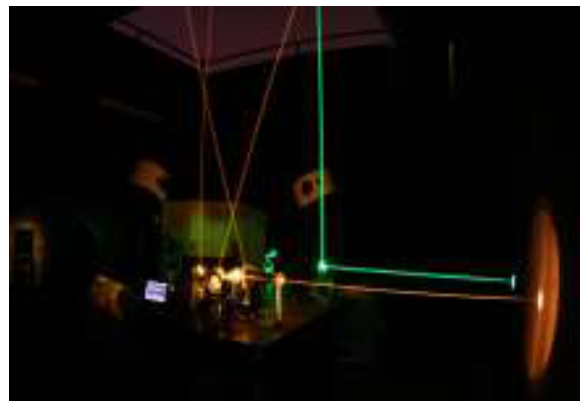

Figure $2589 \mathrm{~nm}$ and $532 \mathrm{~nm}$ laser beams of the transmitter

The frequency of $589 \mathrm{~nm}$ seeder laser is locked by Doppler-free spectroscopy of the sodium D2 line. Then, two acousto-optic modulators are employed to generate three frequencies with a shift of $630 \mathrm{MHz}, 0$ and $-630 \mathrm{MHz}$ to the seeder laser, respectively[11]. The cw seeder laser is amplified by a pulsed dye amplifier. These three frequencies are changed one by one with a period of 30 seconds to measure the number of neutral sodium atoms with three kinds of different velocity, which are sensitive to the wind and temperature.

The optical receiver is combined by three telescopes, two for west and north direction observing with an effective area of about $0.78 \mathrm{~m}^{2}$ each other, and one combined telescope for zenith observing with an effective area of about $1.13 \mathrm{~m}^{2}$. The combined telescope is combined by four telescopes with a diameter of $1.2 \mathrm{~m}$ each other, and will be shared for multi channels. The lidar echoes excited by multi laser beams are focused at different positions of the focal plane of the telescope and received by their own fibers, as shown in figure 3 . This technique has been verified in the optical receiver of the old lidar system in WIPM[14].

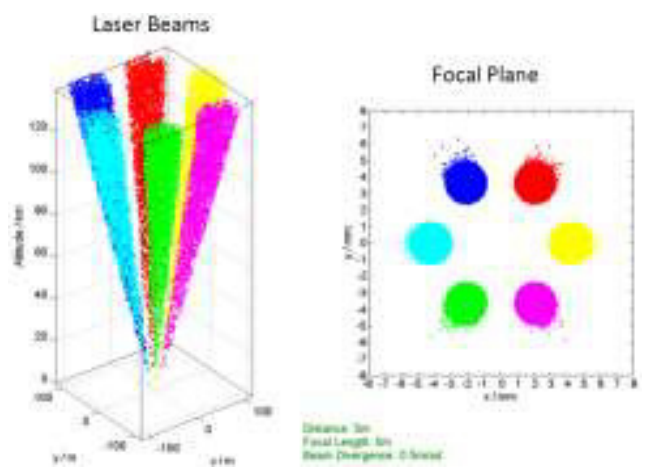

Figure 3 laser beams (left ) and their image (right) in the focal plane of the telescope

The signal processing and data platform records the lidar echoes and processes 16 data sets. One of them is for the Rayleigh temperature channel, two for the rotational Raman channel, four for the Doppler wind channel and nine for the sodium channel. An iodine filter is employed in the Doppler wind channel to discriminate the Doppler shift induced by the atmospheric wind. An interference-filter-based polychromator is used in the rotational Raman channel to block the Rayleigh and Mie scattering and split the Raman scattering echoes with high and low quantum number $\mathrm{J}$ into two photomultiplier tubes. Based on lidar echoes, the data platform retrieves the aerosol backscatter coefficient, atmospheric density in the stratosphere and mesosphere and sodium density in the mesopause. The temperature for altitudes between $5 \sim 40 \mathrm{~km}$ is retrieved within the pure rotational Raman scattering methodology. The temperature in the mesosphere is retrieved based on the ideal gas law and hydrostatic equilibrium assumption. The echoes from the sodium atoms with different velocities of thermal motion are used to calculate the temperature ratio and wind ratio, and therefore to obtain the wind and temperature. The 
relationships between the temperature, wind and their ratios are show in figure 4.

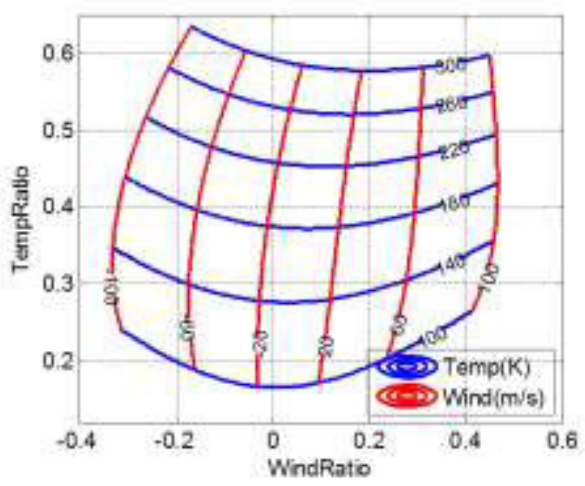

Figure 4 The temperature ratio and wind ratio are used to calculate the temperature and wind

\section{RESULTS}

The new lidar system is still under construction in the laboratory and the telescopes have not been installed so far. However, a small telescope has been used as a substitute to tune and adjust the lidar system. The lidar echoes of all channels have been obtained, and some of them are shown in figure 5 and 6.
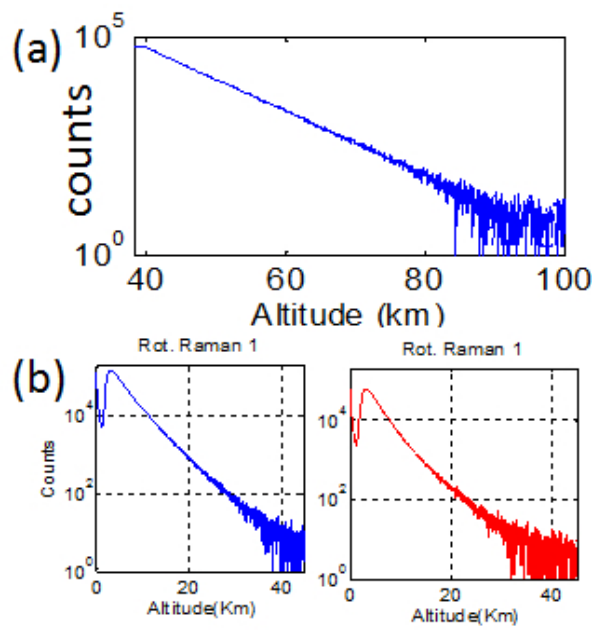

Figure 5 lidar echoes of Rayleigh channel(a) and Raman channel(b)

The atmospheric density, temperature and wind are measured based on the observation of the rotational Raman channel, Rayleigh temperature channel, sodium channel and Doppler wind channel. The vertical profiles of temperature will be shown in this section. Figure 7 shows the temperature in the troposphere and stratosphere observed by the rotational Raman channel with a
55 min temporal resolution and $75 \mathrm{~m}$ spatial resolution. The temperature measured by the lidar (blue solid line in the left panel of figure 7) agrees well with the radiosonde measurements (red circular in the left panel of the figure 7) provided by the Wuhan Weather Station[7].

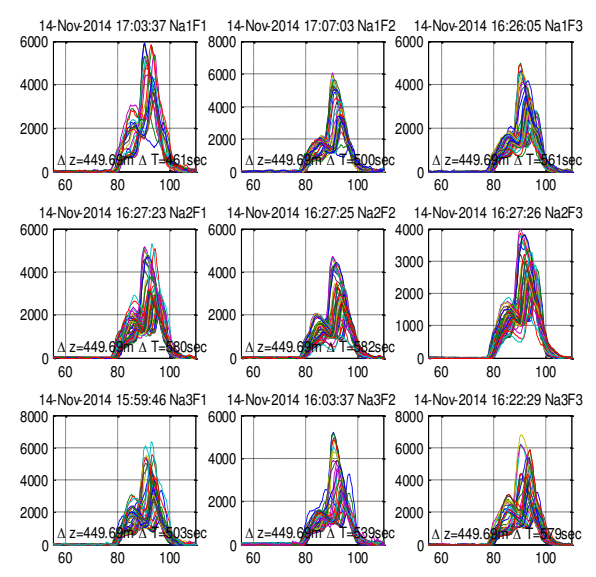

Figure 6 lidar echoes of sodium channel
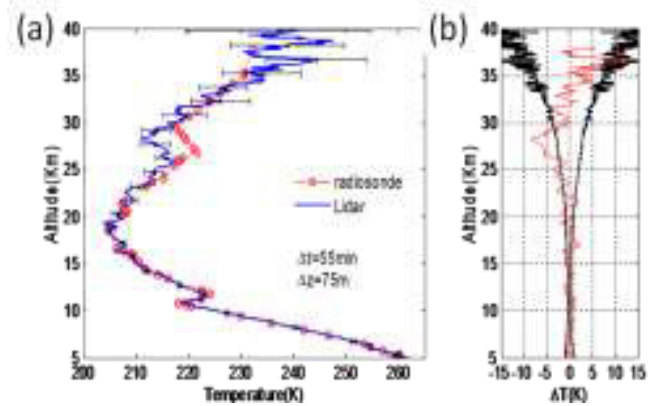

Figure 7 Temperature profile (left) and its error (right) measured by the rotational Raman channel

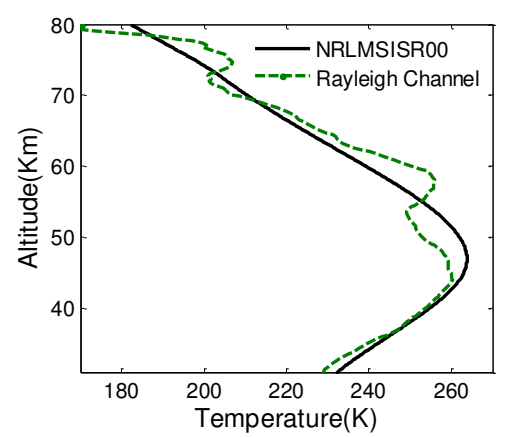

Figure 8 Temperature in the stratosphere and mesosphere measured by the Rayleigh channel

The temperature profile of the stratosphere and mesosphere retrieved from the Rayleigh signal is shown in Figure 8. Figure 9 shows the temperature profile of the mesopause measured by 
the sodium channel. The temporal and spatial resolution are $30 \mathrm{~min}$ and $900 \mathrm{~m}$, respectively. The lidar observed temperature is consistent with the atmospheric model. The measurement precision will be scaled in further data process.

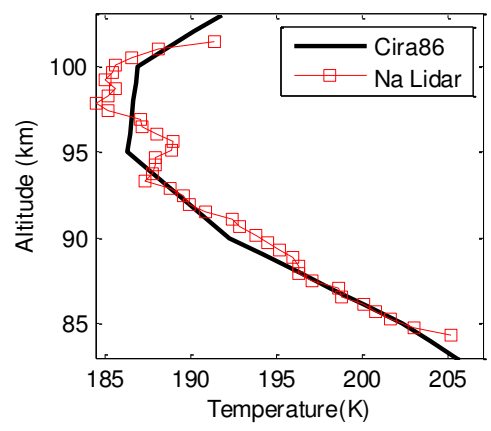

Figure 9 The temperature profile of the mesopause measured by the sodium channel

The temperature results from Raman, Rayleigh and sodium channel will be merged to obtain the whole atmosphere thermodynamic structure. The temperature profiles from Raman and Rayleigh channel have a $\sim 5-\mathrm{km}$ overlap, which could verify the measurement for each other. The combined telescope will be used to enhance the effective area of the optical receiver in the next step. Thus, there would also be an overlap for the temperature profiles measured by the Rayleigh and sodium channel. This lidar system will be installed in two chambers and take part in the field observation.

\section{CONCLUSIONS}

A new lidar system was developed in Wuhan Institute of Physics and Mathematics to observe the atmospheric density, temperature and wind. The design and progress of this lidar system was reported. The atmospheric temperature of the troposphere, stratosphere and mesopause were measured by the Raman, Rayleigh and sodium channel of this lidar respectively.

\section{ACKNOWLEDGEMENT}

The radiosonde data was supported by the Wuhan Weather Station. This work was supported by the National Natural Science Foundation of China, under the Grant nos. 11403085, 41127901, 41101334.

\section{REFERENCES}

[1]. Dunker T, Hoppe U-P, Feng W, Plane JMC, Marsh DR. 2015: Mesospheric temperatures and sodium properties measured with the ALOMAR $\mathrm{Na}$ lidar compared with WACCM.

[2]. Whiteway JA, Carswell AI. 1994: Rayleigh lidar observations of thermal structure and gravity wave activity in the high arctic during a stratospheric warming, $J$ Atmos Sci, 51(21),3122-36.

[3]. Hauchecorne A, Chanin ML, Keckhut P. 1991: Climatology and trends of the middle atmospheric temperature $(33-87 \mathrm{~km})$ as seen by Rayleigh lidar over the south of France, Journal of Geophysical Research: Atmospheres, 96(D8), 15297-309.

[4]. Cooney J. 1972: Measurement of atmospheric temperature profiles by Raman backscatter, J Appl Meteorol, 11(1),108-12.

[5]. Keckhut P, Chanin M, Hauchecorne A. 1990: Stratosphere temperature measurement using Raman lidar, Applied optics, 29(34),5182-6.

[6]. Di Girolamo P. 2004: Rotational Raman Lidar measurements of atmospheric temperature in the UV, Geophysical Research Letters, 31(1).

[7]. YaJuan L, ShaLei S, Faquan L, Xuewu C, Zhenwei C, Linmei L, et al. 2015: High Precision Measurements of lower atmospheric temperature based on Pure Rotational Raman Lidar, submitted to Chinese Journal of Geophysics.

[8]. Chu X, Pan W, Papen GC, Gardner CS, Gelbwachs JA. 2002: Fe Boltzmann temperature lidar: design, error analysis, and initial results at the North and South Poles, Applied Optics, 41(21),4400-10.

[9]. Höffner J, Lautenbach J. 2009: Daylight measurements of mesopause temperature and vertical wind with the mobile scanning iron lidar, Optics Letters, 34(9),1351-3.

[10]. Gibson AJ, Thomas L, Bhattachacharyya SK. 1979: Laser observations of the ground-state hyperfine structure of sodium and of temperatures in the upper atmosphere, Nature, 281.

[11]. She CY, Sherman J, Yuan T, Williams BP, Arnold K, Kawahara TD, et al. 2003: The first 80-hour continuous lidar campaign for simultaneous observation of mesopause region temperature and wind, Geophysical Research Letters, 30(6), 1319.

[12]. Hu XO, Yan ZA, Guo SY, Cheng YQ, Gong JC. 2011: Sodium fluorescence Doppler lidar to measure atmospheric temperature in the mesopause region, Chinese Sci Bull, 56(4-5),417-23.

[13]. Alpers M, Eixmann R, Fricke-Begemann C, Gerding M, Hoeffner J. 2004: Temperature lidar measurements from 1 to $105 \mathrm{~km}$ altitude using resonance, Rayleigh, and Rotational Raman scattering, Atmospheric Chemistry and Physics, 4(3), 793.

[14]. Cheng XW, Gong SS, Li FQ, Dai Y, Song J, Wang JM, et al. 2007: $24 \mathrm{~h}$ continuous observation of sodium layer over Wuhan by lidar, Sci China Ser G, 50(3),287-93. 